\title{
Metabolomics Analysis Reveals the Mechanism of Hydrogen Cyanamide in Promoting Flower Bud Break in Blueberry
}

\author{
Hao Wang, Xiuying Xia * and Lijia An
}

check for updates

Citation: Wang, H.; Xia, X.; An, L. Metabolomics Analysis Reveals the Mechanism of Hydrogen Cyanamide in Promoting Flower Bud Break in Blueberry. Agronomy 2021, 11, 102. https://doi.org/10.3390/agronomy 11010102

Received: 25 November 2020

Accepted: 5 January 2021

Published: 7 January 2021

Publisher's Note: MDPI stays neutral with regard to jurisdictional clai$\mathrm{ms}$ in published maps and institutional affiliations.

Copyright: (C) 2021 by the authors. Licensee MDPI, Basel, Switzerland. This article is an open access article distributed under the terms and conditions of the Creative Commons Attribution (CC BY) license (https:// creativecommons.org/licenses/by/ $4.0 /)$.
School of Bioengineering, Dalian University of Technology, Dalian 116024, China; hao2012wang@163.com (H.W.); bioeng@dlut.edu.cn (L.A.)

* Correspondence: xx47@dlut.edu.cn; Tel.: +86-135-0408-8133

\begin{abstract}
Bud dormancy of deciduous fruit trees is a complex process that allows trees to survive long periods in adverse conditions during winter. Dormancy is a major obstacle for both fruit production in mild winter areas and off-season culture of fruit trees in protection facilities. It is very economically advantageous to be able to control the time point of bud break and consequently harvest in crops with high returns and short harvest seasons like blueberry (Vaccinium spp.). Hydrogen cyanamide $\left(\mathrm{H}_{2} \mathrm{CN}_{2}(\mathrm{HC})\right)$ treatment is an effective method to promote dormancy release and synchronize bud break in perennial deciduous fruit trees, including blueberry. However, there are few systematic studies of the metabolic changes that occur during HC-induced bud breaking. In this study, the metabolome of blueberry buds under forced conditions following HC and water treatment (control) was analyzed using gas chromatography paired with time-of-flight mass spectrometry (GC-TOFMS) technology. A total of 252 metabolites were identified and 16 differential metabolites (VIP $>1, p<0.05$ ) were detected. The levels of several soluble sugars (fructose, glucose, maltose), organic acids (citric acid, alpha-ketoglutaric, succinic acid), and amino acids (aspartic acid, glutamic acid, phenylalanine) were upregulated, while tyrosine, tryptophan, and asparagine were significantly downregulated in HC-treated buds when compared with control buds. The synthesis and accumulation of phenylpropanoids (salicin, 4-vinylphenol, neohesperidin) were also promoted by HC. These results suggest that alteration of carbohydrate and amino acid metabolism, tricarboxylic acid (TCA) cycle increase, and phenylpropanoid accumulation were crucial in HC-promoted bud breaking in blueberry. This research extends our understanding of the mechanisms involved in dormancy release induced by $\mathrm{HC}$ and provides a theoretical basis for applying HC to accelerate bud break.
\end{abstract}

Keywords: blueberry; metabolomics; bud break; hydrogen cyanide; phenylpropanoids

\section{Introduction}

Bud dormancy of deciduous fruit trees is a complex process that allows trees to survive in adverse conditions during winter [1]. Bud dormancy release and therefore bud break is achieved by sufficient exposure to chilling conditions and subsequent warm condition. Warm winters result in reduced and irregular bud break, which consequently lead to reduced fruit production [2]. On the other hand, off-season culture of fruit trees in protection facilities requires controllable and synchronized bud break to obtain more economic benefit.

Blueberry (Vaccinium spp.) is an important perennial fruit crop and is very popular worldwide due to its high antioxidant and anti-inflammatory capacities. In recent decades, blueberry production has expanded rapidly around the world. However, the short and concentrated harvest season of blueberries make it difficult to achieve a year-round supply of fresh fruits. It is very economically advantageous to be able to control the time point of bud break and harvest in crops with high returns like blueberry.

Regulation of bud break is considered to be reliable solution to ensure deciduous fruit production under changing climate conditions as well as in the practice of off-season 
cultivation. Chemicals agents such as gibberellins, abscisic acid, ethylene, hydrogen cyanamide (HC), and thiourea can be utilized to promote dormancy release and bud break. $\mathrm{HC}$ is widely used by growers to overcome low and uneven bud break in deciduous fruit trees, including grape (Vitis vinifera L.) [3], sweet cherry (Prunus avium L.) [4], peach (Prunus persica L.) [5], rabbiteye blueberry (Vaccinium ashei Reade) and southern highbush blueberry (V. corymbosum L.) [6].

Dormancy release and bud break are associated with numerous physiological and biochemical processes that are regulated by gene expression, protein synthesis, hormonal signaling and energy metabolism [7]. Some reports have illustrated that exogenous HC application influences carbohydrate metabolism, respiration and phosphorylation by altering $\alpha$-amylase activity and the expression level of sucrose synthase, pyruvate decarboxylase, sucrose non-fermenting (SNF)-like protein kinase, and alcohol dehydrogenase [8]. Several pieces of evidence indicate that $\mathrm{HC}$ application induces oxidative stress through elevation of production of hydrogen peroxide $\left(\mathrm{H}_{2} \mathrm{O}_{2}\right)$, which is a stimulus of growth initiation [9]. Moreover, exogenous HC application increases ethylene and cytokinin content to promote dormancy release [10]. Recently, transcriptome and proteome analysis have been conducted to investigate the role of HC in grape bud dormancy release. The results show that many genes and proteins involved in metabolic, ribosomal, and hormonal signaling pathways are differentially expressed between HC-treated and control group [11].

Although, worldwide, commercial use of $\mathrm{HC}$ is extensive, the mechanism of action of $\mathrm{HC}$ based on metabolic pathways has not been fully revealed. The development of metabolomics technologies has improved our understanding of the complex molecular interactions of biological systems. Metabolomics is defined as the comprehensive and quantitative analysis of all small molecules in a biological organism [12]. Metabolomics has great potential to substantially improve our understanding of plant-chemical interactions [13]. However, there are only a few metabolomic studies of deciduous fruit trees during dormancy as well as bud break, there is still a lack of data on the relevant metabolites connected with HC-induced bud break, especially the changes of oligosaccharides, amino acids and phenolic compounds.

In this study, gas chromatography-quadrupole-time-of-flight mass spectrometry (GC-TOFMS) based metabolomics analysis was performed to investigate the changes in metabolic profiles induced by exogenous HC application. The purpose of this study was to characterize the differential metabolites involved in HC-promoted bud breaking, and to provide important information for comprehensive understanding of the mechanism of HC-induced budbreak in blueberries.

\section{Materials and Methods}

\subsection{Plant Materials and Chemicals}

Five-year-old trees of northern highbush (Vaccinium corymbosum L.) cultivar 'Bluecrop' were used in this trial. The plants were grown in a commercial blueberry plantation located in Chengzitan town $\left(39.53^{\circ} \mathrm{N}, 122.41^{\circ} \mathrm{E}\right.$, altitude $\left.25 \mathrm{~m}\right)$, Dalian, Liaoning Province, China.

\subsection{HC Treatment and Sampling}

On 28 December 2016 (according to our production experience, blueberry buds were in endodormancy-ecodormancy stage during this period), branches with 4-5 flower buds were randomly collected from trees. The branches were divided into two groups; one group was sprayed to the point of run off with an aqueous solution of Dormex ${ }^{\circledR}$ (Ningxia Darong Chemicals \& Metallurgy Co., Ltd., Ningxia, China), containing $0.67 \%$ (v/v) HC. The other group was sprayed with distilled water to serve as a control. After treatment, the branches were placed in water in vials in a growth chamber $\left(23 \pm 2{ }^{\circ} \mathrm{C} ; 12 \mathrm{~h}\right.$ light: $12 \mathrm{~h}$ dark photoperiod; 75\% humidity). The water in the vials was changed, and the basal ends of the branches were cut every $2-3 \mathrm{~d}$. The percentage of bud break was measured to determine the dormancy status. Buds were sampled 7 days after HC application. On this time point, the bud break rate of the HC-treated branches is higher than $50 \%$, and the bud 
break rate of the control is lower than $50 \%$. To eliminate the bias of natural developmental heterogeneity, the flower buds with different developmental stages of one entire branch were combined as samples of each biological replicate. The samples were immediately frozen in liquid nitrogen and stored at $-80^{\circ} \mathrm{C}$ until analysis.

\subsection{Metabolite Extractions}

Fifty milligrams of bud samples from each group $(N=5)$ was transferred into a $2 \mathrm{~mL}$ microcentrifuge tube containing $0.4 \mathrm{~mL}$ of water/methanol $(1: 3 v / v)$. Then, $20 \mu \mathrm{L}$ of adonitol $\left(1 \mathrm{mg} / \mathrm{mL}\right.$ stock in $\left.\mathrm{dH}_{2} \mathrm{O}\right)$ was added to each sample as an internal standard. The samples were homogenized with a ball mill (Retsch Corporation, Haan, Germany) for $4 \mathrm{~min}$ and ultrasonicated at $60 \mathrm{~Hz}$ for $5 \mathrm{~min}$ (incubated in ice water). The samples were centrifuged for $15 \mathrm{~min}$ at $13,000 \mathrm{rpm}, 4{ }^{\circ} \mathrm{C}$. A $300 \mu \mathrm{L}$ aliquot of supernatant was transferred to a glass vial for vacuum-drying at room temperature; $50 \mu \mathrm{L}$ of methoxylamine hydrochloride $(20 \mathrm{mg} / \mathrm{mL}$ in pyridine) was then added. The samples were incubated for $30 \mathrm{~min}$ at $80{ }^{\circ} \mathrm{C}$. About $70 \mu \mathrm{L}$ of $\mathrm{N}, \mathrm{O}$-bis(trimethylsilyl)trifluoroacetamide (BSTFA) reagent ( $1 \%$ trimethyl chlorosilane, $v / v$ ) was then added, and the sample was vortexed vigorously for $2 \mathrm{~min}$ prior to derivatization at $70{ }^{\circ} \mathrm{C}$ for $2 \mathrm{~h}$. All samples were analyzed by GC-TOFMS.

\subsection{GC-TOFMS Analysis}

GC-TOFMS analysis was performed using an Agilent 7890 gas chromatograph system coupled with a Pegasus HT time-of-flight mass spectrometer (Agilent Corporation, Santa Clara, CA, USA). The system utilized a DB-5MS capillary column coated with 5\% diphenyl cross-linked with 95\% dimethylpolysiloxane $(30 \mathrm{~m} \times 250 \mu \mathrm{m}$ inner diameter, $0.25 \mu \mathrm{m}$ film thickness; J\&W Scientific, Folsom, CA, USA). Briefly, each $1 \mu \mathrm{L}$ aliquot of the derivatized sample was injected in splitless mode. Helium was used as the carrier gas, the front inlet purge flow was $3 \mathrm{~mL} \mathrm{~min}{ }^{-1}$, and the gas flow rate through the column was $1 \mathrm{~mL} \mathrm{~min}^{-1}$. The initial temperature was maintained at $50{ }^{\circ} \mathrm{C}$ for $1 \mathrm{~min}$, then raised to $310^{\circ} \mathrm{C}$ at a rate of $10^{\circ} \mathrm{C} \mathrm{min}-1$, then maintained at $310^{\circ} \mathrm{C}$ for $8 \mathrm{~min}$. The injection, transfer line, and ion source temperatures were 280,270 , and $220^{\circ} \mathrm{C}$, respectively. The energy was $-70 \mathrm{eV}$ in electron impact mode. Mass spectrometry data were acquired in full-scan mode with the $\mathrm{m} / \mathrm{z}$ range of 50-500 at a rate of 20 spectra per second after a solvent delay of $366 \mathrm{~s}$.

Chroma TOF 4.3X software of LECO Corporation and LECO-Fiehn Rtx 5 database (LECO Corporation, St. Joseph, MI, USA) were used for raw peak extraction, baseline filtering and calibration, peak alignment, deconvolution analysis, peak identification, and integration of the peak area. The RI (retention time index) method was used for peak identification, and the RI tolerance was 5000. Metabolites were quantified by the peak area ratio of each peak and internal standard.

After obtaining the collated data, we performed a series of multivariate pattern recognition analyses, and principal component analysis (PCA) was first used to investigate the overall blueberry bud metabolome. The SIMCA-P14.1 software package (V14.1, MKS Data Analytics Solutions, Umea, Sweden) was used to perform logarithmic conversions and centralization formatting of the data, follow by automated modeling analysis. In order to filter out the orthogonal variables in the metabolite that were not related to the categorical variables and to obtain more reliable metabolite information on the differences between groups, orthogonal projections to latent structures discriminant analysis (OPLS-DA) was applied to extract maximum information from the dataset and to analyze the results. In this mode, the remaining variables were then screened by Student's $t$-test $(p$-value $<0.05)$ and the variable importance in the projection (VIP $>1$ ) of the first principal component of the OPLS-DA model.

\section{Results}

\subsection{Bud Break Rate after HC Treatment}

Branches of blueberry were cultured in a growth chamber. The buds were treated with $\mathrm{HC}$, and the percentage of bud break was determined up to 13 days after treatment 
(DAT). The application of HC to dormant blueberry buds advanced bud break. As shown in Figure 1, the bud breaking rate of the control branch with water treatment was less than $50 \%$ within 10 DAT. On day $7,79.3 \%$ of the HC treatment group underwent bud break, compared with $34.4 \%$ in the control group. On the 13th day, the bud breaking rate of the control group was $76 \%$, which was still lower than $90 \%$ for the HC treatment group.

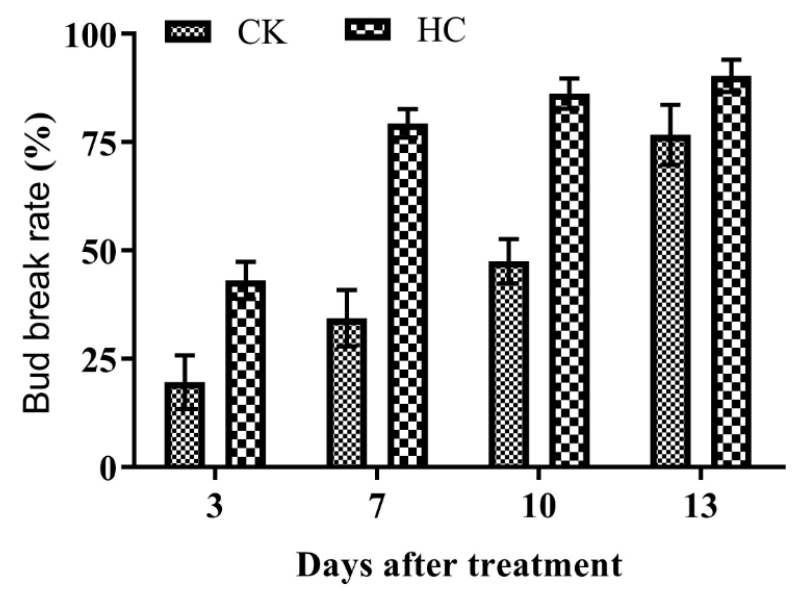

Figure 1. Bud break responses of the $\mathrm{HC}$ treatment $(\mathrm{HC})$ and the control $(\mathrm{CK})$ at $3,7,10$, and 13 days after treatment. Bars represent \pm SEM of three biological replicates.

\subsection{Global Metabolic Response of Blueberry Buds after HC Treatment}

The metabolite profiles of blueberry buds were obtained via GC-TOFMS-based metabolomics. A total of 672 peaks were detected, and 252 metabolites were identified using the BinBase identifier on the basis of the retention index and MS fingerprint matches. In order to investigate whether $\mathrm{HC}$ treatment regulated the entire metabolic profile of buds, we conducted a multivariate statistical analysis using unsupervised principal component analysis (PCA) and supervised orthogonal partial least squares discrimination analysis (OPLS-DA). The PCA scores plot (Figure 2A) showed that HC treatment was clearly separated from the control along the first principal component (PC1), which accounted for $73.5 \%$ of the total variance. OPLS-DA data analysis (Figure 2B) also confirmed the ability to separate HC-treated samples from the controls through a predictive model that was statistically significant $\left(\mathrm{R}^{2}=0.979, \mathrm{Q}^{2}=-0.478\right)$ (Figure $\mathrm{S} 1$ ). These results suggest that HC treatment alters the metabolic profile of blueberry buds.

A

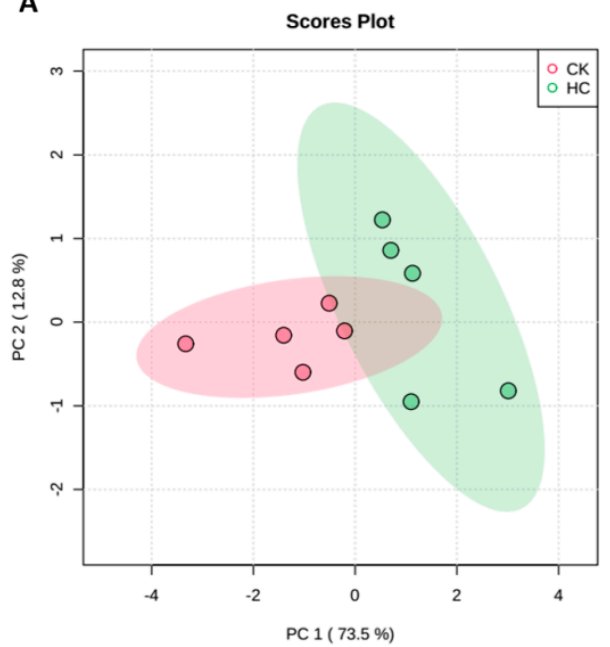

B

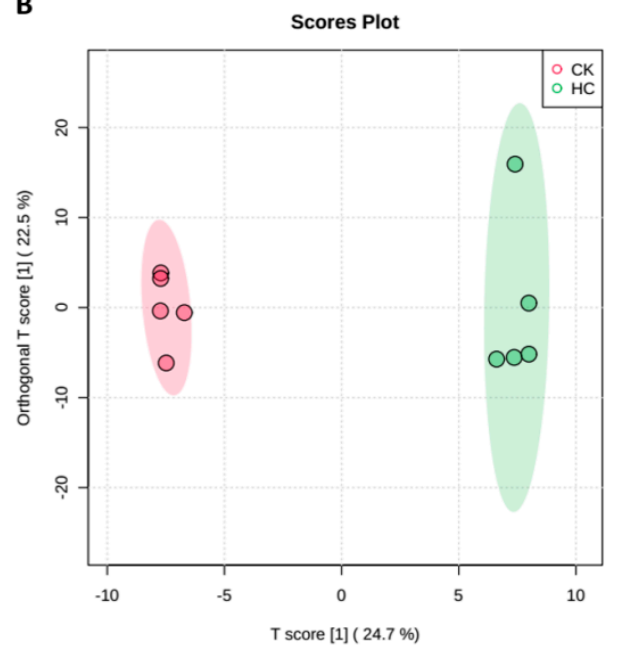

Figure 2. PCA (A) and OPLS-DA (B) score plots of metabolic profiles in the HC treatment (HC) and the control (CK). 


\subsection{Changes in Metabolite Levels after HC Treatment}

According to Student's $t$-test, 49 metabolites were selected from 252 metabolites with $p$ values $<0.05$. Hierarchical cluster analysis of these features helped us to classify metabolites and uncover the changing characteristics of the metabolites in the experimental group (Figure S2). Metabolites were divided into sugar, amino acids, amino acid derivatives, organic acids, phenylpropanoids, and other compounds. Among the 49 identified metabolites, 10 were downregulated and 39 were upregulated in the HC treatment group compared with the control treatment group. The level of most sugars, amino acids, and organic acids tended to increase in blueberry buds after HC treatment. Furthermore, 16 different metabolites were selected according to their VIP values in the OPLS-DA model $(\mathrm{VIP}>1)$ and $p$-values from Student's $t$-test $(p<0.05)$ in Table 1 . The two metabolites with the highest VIP value are oxoproline (7.93) and glutamine (5.14). Asparagine and citric acid are the two metabolites with the greatest difference, and their fold change (FC) values are -29.39 and 28.58 , respectively.

Table 1. List of the 16 metabolites with the most significant differentially quantified between the $\mathrm{HC}$ treatment $(\mathrm{HC})$ and the control (CK). Criteria was highest VIP values and significant $p$-value at $0.05(\mathrm{P})$, including the fold change (FC).

\begin{tabular}{cccc}
\hline \multirow{2}{*}{ Metabolites } & \multicolumn{3}{c}{ HC vs. CK } \\
\cline { 2 - 4 } & VIP & $\mathbf{P}$ & FC \\
\hline Oxoproline & 7.93 & 0.004 & 1.15 \\
Glutamine & 5.14 & $<0.001$ & 2.11 \\
Serine & 2.47 & $<0.001$ & 1.49 \\
Alanine & 2.30 & 0.004 & 1.61 \\
Isoleucine & 1.84 & $<0.001$ & 1.83 \\
Asparagine & 1.77 & $<0.001$ & -29.39 \\
Glutamic acid & 1.75 & 0.001 & 1.50 \\
Citrulline & 1.52 & 0.001 & 2.43 \\
Allothreonine & 1.35 & $<0.001$ & 1.52 \\
Galactinol & 2.76 & 0.035 & -1.72 \\
Myo-inositol & 1.33 & 0.010 & 0.69 \\
Succinic acid & 1.79 & 0.013 & 0.72 \\
Citric acid & 1.41 & 0.041 & 28.58 \\
Phosphate & 1.71 & 0.005 & 0.72 \\
Salicin & 1.63 & $<0.001$ & 2.09 \\
Maltotriose & 1.04 & $<0.001$ & -2.48 \\
\hline
\end{tabular}

$\overline{\text { VIP (variable importance in the projection) values were obtained from OPLS-DA models with a threshold of } 1 .}$ $p$ values were calculated from student's t-test with a threshold of 0.05 . FC (fold change) values were obtained from mean peak area of the former group/mean peak area of the latter group. If the FC value is greater than 0 , it means that metabolite level was higher in the former compared with the latter group. 


\subsection{Effects of HC Treatment on Carbohydrate Metabolism and TCA Cycle of Blueberry Buds}

In most plants, carbohydrates represent the major energy store as well as the building blocks of essential structural polymers [14]. The tricarboxylic acid (TCA) cycle is a key metabolic pathway in plants that is linked with energy metabolism and is responsible for the oxidation of respiratory substrates to drive ATP synthesis. Energy metabolism is one of the key factors connected with the release of bud dormancy [15]. The intermediates of glycolysis and the TCA cycle are crucial precursors involved in several metabolic pathways, such as amino acid metabolism and biosynthesis of plant secondary metabolites. As shown in Figure 3, the level of fructose, glucose, and maltose were significantly upregulated in HC-treated buds compared with control buds, whereas the level of maltotriose was significantly downregulated. In addition, the levels of most metabolites involved in the TCA cycle, such as citric acid, fumaric acid, succinic acid, and alpha-ketoglutaric acid, were significantly upregulated in blueberry buds in response to HC treatment. Our results showed that the increase of hexose content after HC treatment may promote activation of the Embden-Meyerhof-Parnas (EMP) pathway. The intermediate products of the EMP pathway provide precursors for many amino acids. At the same time, the TCA cycle was increased, which was beneficial to promoting bud germination.

Among the various metabolic networks, amino acid metabolism gained considerable interest. Amino acids are not only used to synthesize proteins, but also serve as precursors for a large array of metabolites with multiple functions in plant growth and response to various stresses. We observed that the level of amino acids which are made from oxaloacetate (aspartate, homoserine, and threonine) and alpha-ketoglutaric acid (glutamate, glutamine, oxoproline, and citrulline) were significantly upregulated in buds of the HCtreated group compared with the control group; however, the level of asparagine was significantly downregulated. The branched-chain amino acids isoleucine and valine along with alanine and serine were significantly upregulated in buds of the HC-treated group. The aromatic amino acid phenylalanine was significantly upregulated in buds of the HCtreated group compared with the control group, while tyrosine and tryptophan were significantly downregulated (Figure 3). The increase in amino acids can provide substrates and nutrition for bud germination and increase the germination rate.

\subsection{Effects of HC Treatment on Phenylpropanoid Metabolites of Blueberry Buds}

Phenylpropanoid metabolism is an important pathway for the synthesis of secondary metabolites. Phenylpropanoids are a variety of organic compounds that are synthesized by plants from phenylalanine. As shown in Figure 3, phenylalanine was significantly enriched in HC-treated buds, and the relative abundance of salicin, 4-vinylphenol, vanillic acid, 3-methylcatechol, and neohesperidin increased significantly. The levels of epigallocatechin and sinapinic acid were significantly downregulated in blueberry buds in response to HC treatment. Many phenylpropanoids possess strong antioxidant properties against free radicals that caused by various stress $[16,17]$. HC promoted the phenylpropanoid metabolism pathway and harmonized phenylpropanoid synthesis, which may contribute to the induction of antioxidant defense mechanism and increase the bud break rate. 


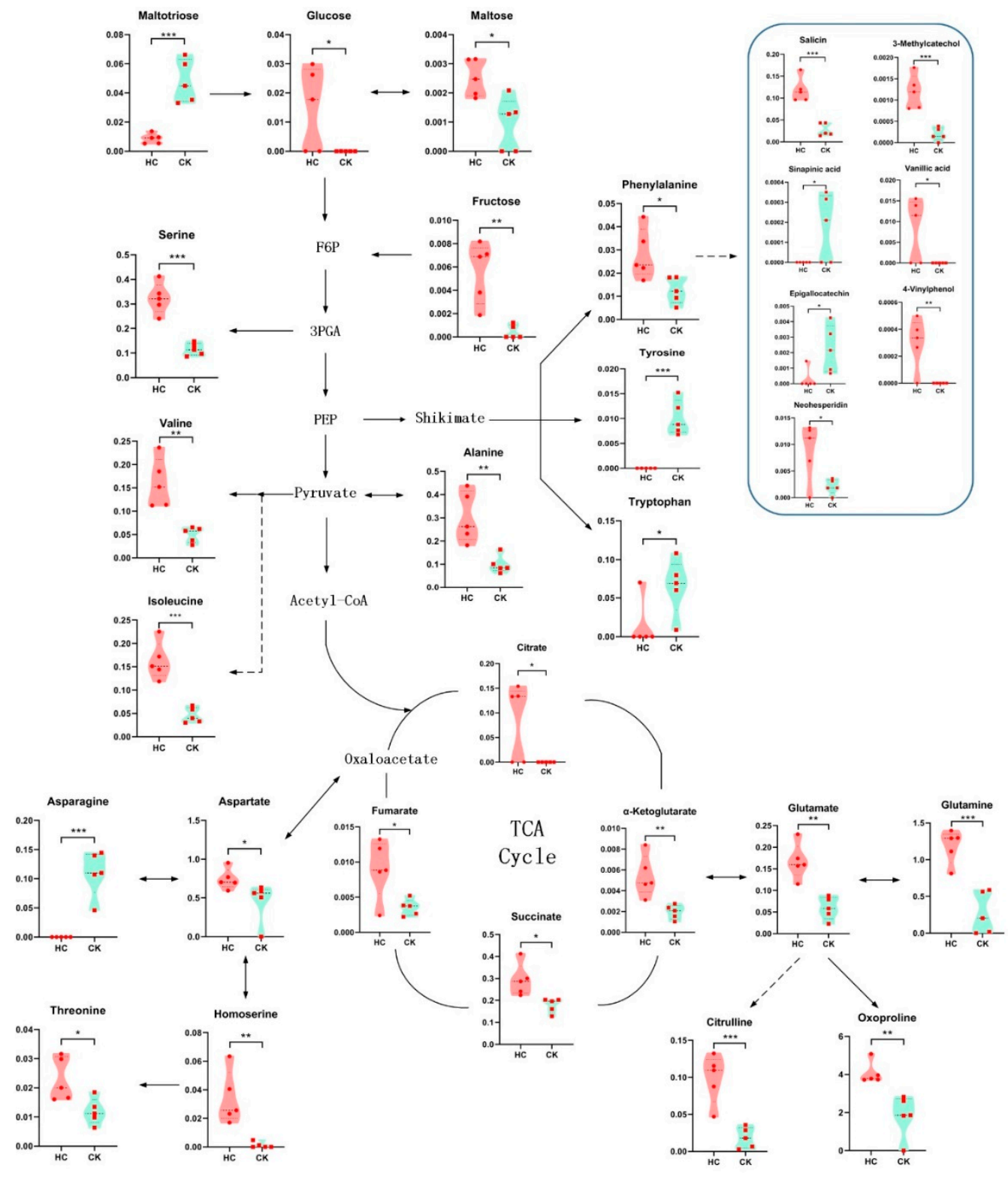

Figure 3. Changes in metabolites of the metabolic pathways in the HC treatment (HC) and the control (CK). The values on the Y-axis indicate the relative content of metabolite. "**, “***, and "***” represent significant differences between the HC treatment (HC) and the control (CK) at the $p<0.05, p<0.01$, and $p<0.001$ levels.

\section{Discussion}

$\mathrm{HC}$ is a frequently used agrochemical that successfully advances bud break in a variety of fruit trees. In this study, compared with the control treatment, the application of HC in the dormancy phase resulted in earlier bud break and higher bud break percentage in blueberries (Figure 1), as expected, which is similar to the results found in rabbiteye and southern highbush blueberry [6]. Furthermore, after HC treatment, the bud development state changed, and the contents of many primary and secondary metabolites were also significantly altered (Figure 3).

Carbohydrate metabolism plays an important role in regulating dormancy release processes in plants. Carbohydrate reserves are the main source of energy for the metabolic changes that occur during the dormant and budburst period [18]. Fernandez et al. (2019) found that concentrations of starch and hexoses are closely related with dormancy progression. Previous studies in deciduous fruit trees indicated that starch concentration in twigs 
decreased as chill accumulated in the orchard, while concentrations of hexoses (glucose and fructose) increased. Starch and hexoses concentrations are physiological markers in the dormancy progression of sweet cherry (Prunus avium L.) [19]. Previous studies have reported that the increase in bud break percentage in response to $\mathrm{HC}$ application was accompanied by significant variations in carbohydrate metabolism of buds [20]. HC treatment influences the expression of genes and proteins associated with carbohydrate metabolism [18], and causes an increase in soluble sugars accumulation and a decrease in starch content in grape [3]. Soluble sugar not only provides energy for plant growth but also acts as a signal molecule to regulate plant growth [18]. Glucose and fructose might also serve as a signaling molecule promoting bud outgrowth [21]. In our study, HC treatment significantly increased the levels of fructose, glucose, and maltose and decreased maltotriose level (Figure 3). This result is supportive of previous findings. We speculate that HC promote bud dormancy release in blueberry by affecting carbohydrate metabolism. $\mathrm{HC}$ may promote the hydrolyzation of starch and oligosaccharides and increase soluble sugar content, thereby providing signals and more energy for the buds to resume growth.

The TCA cycle is the center of material and energy metabolism. Previous researchers found that the TCA cycle was increased during buds dormancy release in numerous species including peach [22], peony (Paeonia suffruticosa cv. Luhehong) [23], and sweet cherry [24]. Horikoshi et al. (2018) also suggested that the pathway involved in carbohydrate metabolism and ATP production in the TCA cycle was stimulated at the end of endodormancy to produce energy for pear bud sprouting [25]. Lisa et al. (2019) reported that genes encoding enzymes involved in the TCA cycle were upregulated by HC treatment in the buds of peach trees [22]. Consistent with these results, our study showed that the levels of most metabolites involved in the TCA cycle such as citric acid, fumaric acid, succinic acid, and alpha-ketoglutaric acid, were significantly higher in HC-treated buds than control buds (Figure 3). These results suggest that HC treatment positively affects the TCA cycle for efficient energy production, which may contribute to earlier bud break and higher bud break percentages in blueberry.

In addition to being incorporated into proteins, plant amino acids serve as precursors for the biosynthesis of secondary metabolites, and their levels change in response to different chemical agents or environmental conditions [26]. Amino acid metabolism is tightly linked to energy and carbohydrate metabolism, the carbon-nitrogen budget, hormones, and stress responses, among other factors [27]. Transition from dormancy to growth is accompanied by changes in the amino acid composition of buds [28]. We observed that the level of most amino acids in the HC-treated buds was significantly increased compared with the control buds (Figure 3). In response to specific developmental stages and stressassociated conditions, amino acids serve as energy donors through catabolic processes [29]. The amino acids produced by protein degradation can be converted into one of the TCA cycle intermediates, such as oxaloacetate or alpha-ketoglutaric acid. We found that alphaketoglutaric acid levels in HC-treated buds clearly increased (Figure 3). The results imply that HC-activated amino acids synthesis and accumulation provide substrates and energy for bud growth and development.

Glutamate, glutamine, aspartate and asparagine are the central regulators of carbon/nitrogen metabolism and they interact with multiple metabolic networks [30,31]. Glutamine and glutamate are the precursors of nitrogenous compounds in plants. The production of glutamate is a key point in the synthesis of a variety of organic molecules, such as nucleic acids, amino acids and secondary metabolites [30]. The glutamate metabolism pathway is strongly associated with proline, oxoproline, and citrulline. Horikoshia et al. (2018) suggested that thermal fluctuation treatment leads to low bud burst percentages and low glutamate and oxoproline levels in pear flower buds [25]. Contrary to the effect of thermal fluctuation treatment, HC treatment increased glutamine, glutamate, oxoproline and citrulline levels in blueberry buds (Figure 3). Asparagine is also an important nitrogen storage and transport molecule in many plant species due to its relatively high nitrogen-carbon ratio [32], and it plays a key role in regulating nitrogen assimilation and 
circulation in plants. Homoserine and threonine are derived from the aspartate pathway. We also found that after HC treatment, asparagine levels decreased significantly, while aspartate, threonine, and homoserine levels increased significantly. This may imply that HC promoted bud break and activated the glutamate metabolism pathway, also in addition to promoting the transformation of asparagine to aspartate and transformation of aspartate into homoserine and threonine.

The branched-chain amino acids (BCAAs) isoleucine, valine and leucine and their derivatives play a key role in regulating cell osmosis, reducing ROS damage to cells, preventing membrane damage during cell expansion, and stabilizing proteins and enzymes [33]. Peng et al. (2015) revealed that BCAA catabolism also provides an alternative energy source under long-term dark treatment in plants [34]. BCAA overaccumulation causes reorganization of the actin cytoskeleton and actin-associated endomembranes in Arabidopsis [27]. Galili et al. (2016) showed that isoleucine is a well-documented electron donor that feeds into the TCA cycle to generate the cellular energy to support bud development and germination [29]. In this study, the level of isoleucine and valine increased in HC-treated buds, suggesting that HC might activate BCAA accumulation to promote bud break.

In plants, serine is predominantly generated during the overall process of photorespiration [35]. Timm (2013) suggested that serine acts as a metabolic signal for the transcriptional regulation of photorespiration, particularly in glycine-to-serine interconversion reactions [36]. In our study, serine levels significantly increased after $\mathrm{HC}$ treatment compared with the control group, which indicated that HC might influence photorespiration.

The aromatic amino acids phenylalanine, tyrosine, and tryptophan are not only essential components of protein synthesis but are also used as precursors for numerous metabolites, such as hormones, cell wall components, and a large number of multifunctional secondary metabolites [37]. They play a pivotal role in the regulation of plant growth and development as well as defense responses [38]. Our data show that in HC-treated buds, phenylalanine levels increased, while tyrosine and tryptophan levels decreased significantly compared with control buds. Phenylalanine is a substrate for the phenylpropanoid pathway, which produces a wide range of antioxidative metabolites. Aromatic amino acids are synthesized from chorismate through shikimate pathway. We speculate that $\mathrm{HC}$ treatment promotes shikimate pathway to shift to phenylalanine synthesis instead of tyrosine and tryptophan synthesis. HC induced bud break synchronously stimulated phenylalanine synthesis and accumulation, and further activated the phenylpropanoid pathway to generate antioxidative metabolites.

Secondary metabolites have a significant effect on many processes of plant growth and development. However, the changes and functions of secondary metabolites in HCinduced blueberry dormancy release are still poorly understood. The phenylpropanoid pathway generates numerous phenolic compounds which are well known to possess antioxidant properties against free radicals caused by stresses [39,40]. Oxidative stresses, are imperative components of the dormancy release and bud break process [11]. Notably, many studies have shown that HC application can trigger transient oxidative stress and activate detoxification systems [41]. The study on dormancy process of raspberry showed that the bud break was consistent with the improvement of antioxidant defense system [42]. Experiments have confirmed that to alleviate oxidative stress induced by $\mathrm{HC}$, the activity of antioxidant enzyme along with key genes involved in protective enzyme metabolism are upregulated $[11,22,43]$. HC can also upregulate genes involved in ascorbate and glutathione detoxification pathways [17,44]. In this work, considerable increases in the levels of phenylpropanoid metabolites, including salicin, 4-vinylphenol, vanillic acid, 3-methylcatechol, and neohesperidin, were observed in HC-treated buds. Nevertheless, the levels of epigallocatechin and sinapinic acid were significantly downregulated in HCtreated buds. We speculate that HC treatment induces higher phenylpropanoid pathway activity and adjusts the balance of phenylpropane metabolism, then leads to some specific phenylpropanoids accumulation, which ultimately causes an increase in antioxidant capac- 
ity. The phenylpropanoid metabolites might be essential for the dormancy maintenance and bud break process. To the best of our knowledge, this is the first time that the role of aromatic amino acids and phenylpropanoid metabolites have been described in the bud breaking process induced by $\mathrm{HC}$.

As shown in Figure 4, we proposed a working model of how HC promotes bud break in blueberry according to the metabolomics analysis. After treatment with HC, the hexose content increased. The EMP pathway was activated and TCA cycle activity increased, which provided the energy for bud break. In addition, the EMP pathway and TCA cycle can provide precursors for amino acid synthesis, increasing the levels of a variety of amino acids used to synthesize proteins and provide nutrition for bud germination. With the increase of phenylalanine, the further synthesis of phenylpropanoids plays an important role in promoting bud break. The accumulation of phenylpropanoids might induce antioxidant defense mechanism. What needs to be pointed out is that the presented model was generated using only one time point of metabolic sampling. At this sampling time, the HC-treated samples had reached the bud break stage (bud break rate is greater than $50 \%$ ); while the control samples had not yet reached this stage (bud break rate is less than $50 \%$ ). Furthermore, the difference of bud break rate between the two samples was the biggest. Therefore, the metabolites changes induced by HC treatment could be truly displayed at this sampling time. The model presented in this study can provide us important clues to explore the mechanism of HC promoting bud break. This research extends on our understanding of the dormancy mechanisms in blueberry buds and provides a theoretical basis for applying HC to breaking bud dormancy. Nevertheless, the detection of metabolites at just one time point cannot directly show us the dynamic response of the sample to external stimuli, the more comprehensive information should be provided in the future research.

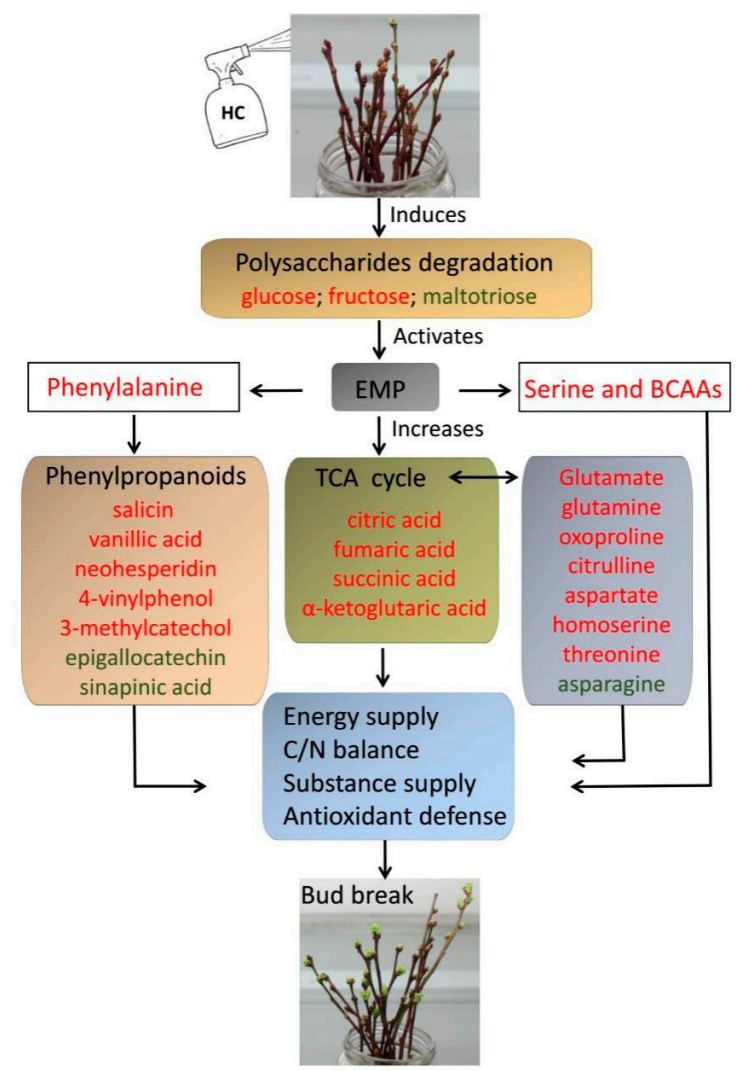

Figure 4. Schematic representation of the mechanisms of blueberry bud break after HC treatment. Red/green indicates metabolites whose relative content increases/decreases after HC treatment compared with CK. 
Supplementary Materials: The following are available online at https:/ /www.mdpi.com/2073-439 5/11/1/102/s1, Figure S1: Permutation test of OPLS-DA model for group HC vs. CK. Figure S2: Heatmap analysis combined with hierarchical cluster analysis (HCA) of the 49 metabolites.

Author Contributions: H.W., X.X. and L.A. conceived and designed the research. H.W. performed the research. H.W. interpreted the experimental data and drafted the manuscript. H.W. and X.X. revised the paper and approved the final version. L.A. and X.X. provided the financial support for this study. All authors have read and agreed to the published version of the manuscript.

Funding: This research received no external funding.

Institutional Review Board Statement: Not applicable.

Informed Consent Statement: Not applicable.

Data Availability Statement: The data presented in this study are available on request from the corresponding author.

Acknowledgments: The authors would like to thank all of the individuals that were involved in this study.

Conflicts of Interest: The authors declare no conflict of interest.

\section{References}

1. Singh, R.K.; Svystun, T.; AlDahmash, B.; Jonsson, A.M.; Bhalerao, R.P. Photoperiod- and temperature-mediated control of phenology in trees-A molecular perspective. New Phytol. 2017, 213, 511-524. [CrossRef] [PubMed]

2. Atkinson, C.J.; Brennan, R.M.; Jones, H.G. Declining chilling and its impact on temperate perennial crops. Environ. Exp. Bot. 2013, 91, 48-62. [CrossRef]

3. Liang, D.; Huang, X.; Shen, Y.; Shen, T.; Zhang, H.; Lin, L.; Wang, J.; Deng, Q.; Lyu, X.; Xia, H. Hydrogen cyanamide induces grape bud endodormancy release through carbohydrate metabolism and plant hormone signaling. BMC Genom. 2019, $20,1034$. [CrossRef] [PubMed]

4. Ionescu, I.A.; Lopez-Ortega, G.; Burow, M.; Bayo-Canha, A.; Junge, A.; Gericke, O.; Moller, B.L.; Sanchez-Perez, R. Transcriptome and metabolite changes during hydrogen cyanamide-induced floral bud break in sweet cherry. Front. Plant Sci. 2017, 8, 1233. [CrossRef] [PubMed]

5. Chen, C.; Beckman, T.G. Effect of a late spring application of hydrogen cyanamide on high-chill peaches. Agronomy 2019, 9, 726. [CrossRef]

6. Williamson, J.G.; Maust, B.E.; Nesmith, D.S. Timing and concentration of hydrogen cyanamide affect blueberry bud development and flower mortality. HortScience 2001, 36, 922-924. [CrossRef]

7. Shangguan, L.; Chen, M.; Fang, X.; Xie, Z.; Gong, P.; Huang, Y.; Wang, Z.; Fang, J. Comparative transcriptome analysis provides insight into regulation pathways and temporal and spatial expression characteristics of grapevine (Vitis vinifera) dormant buds in different nodes. BMC Plant Biol. 2020, 20, 390. [CrossRef] [PubMed]

8. Sudawan, B.; Chang, C.S.; Chao, H.F.; Ku, M.S.; Yen, Y.F. Hydrogen cyanamide breaks grapevine bud dormancy in the summer through transient activation of gene expression and accumulation of reactive oxygen and nitrogen species. BMC Plant Biol. 2016, 16, 202. [CrossRef]

9. Oracz, K.; El-Maarouf-Bouteau, H.; Bogatek, R.; Corbineau, F.; Bailly, C. Release of sunflower seed dormancy by cyanide: Cross-talk with ethylene signalling pathway. J. Exp. Bot. 2008, 59, 2241-2251. [CrossRef]

10. Shi, Z.; Halaly-Basha, T.; Zheng, C.; Weissberg, M.; Ophir, R.; Galbraith, D.W.; Pang, X.; Or, E. Transient induction of a subset of ethylene biosynthesis genes is potentially involved in regulation of grapevine bud dormancy release. Plant Mol. Biol. 2018, 98, 507-523. [CrossRef]

11. Khalil-Ur-Rehman, M.; Wang, W.; Dong, Y.; Faheem, M.; Xu, Y.; Gao, Z.; Shen, Z.G.; Tao, J. Comparative transcriptomic and proteomic analysis to deeply investigate the role of hydrogen cyanamide in grape bud dormancy. Int. J. Mol. Sci. 2019, 20, 3528. [CrossRef] [PubMed]

12. Pasamontes, A.; Cheung, W.H.K.; Simmons, J.; Aksenov, A.A.; Peirano, D.J.; Grafton-Cardwell, E.E.; Kapaun, T.; Dandekar, A.M.; Fiehn, O.; Davis, C.E. Citrus tristeza virus infection in sweet orange trees and a mandarin $\times$ tangor cross alters low molecular weight metabolites assessed using gas chromatography mass spectrometry (GC/MS). Metabolomics 2016, 12, 41. [CrossRef]

13. Luo, Q.; Wang, S.; Sun, L.-n.; Wang, H. Metabolic profiling of root exudates from two ecotypes of Sedum alfredii treated with Pb based on GC-MS. Sci. Rep. 2017, 7, 39878. [CrossRef] [PubMed]

14. Fettke, J.; Fernie, A.R. Intracellular and cell-to-apoplast compartmentation of carbohydrate metabolism. Trends Plant Sci. 2015, 20, 490-497. [CrossRef] [PubMed]

15. Chen, C.; Zeng, L.; Zhao, H.; Ye, Q. Proteomic analysis of the early development of the phalaenopsis amabilis flower bud under low temperature induction using the iTRAQ/MRM approach. Molecules 2020, 25, 1244. [CrossRef] 
16. Farokhzad, A.; Nobakht, S.; Alahveran, A.; Sarkhosh, A.; Mohseniazar, M. Biochemical changes in terminal buds of three different walnut (Juglans regia L.) genotypes during dormancy break. Biochem. Syst. Ecol. 2018, 76, 52-57. [CrossRef]

17. Sayyari, M.; Babalar, M.; Kalantari, S.; Martínez-Romero, D.; Guillén, F.; Serrano, M.; Valero, D. Vapour treatments with methyl salicylate or methyl jasmonate alleviated chilling injury and enhanced antioxidant potential during postharvest storage of pomegranates. Food Chem. 2011, 124, 964-970. [CrossRef]

18. Khalil-Ur-Rehman, M.; Wang, W.; Xu, Y.S.; Haider, M.S.; Li, C.X.; Tao, J.M. Comparative study on reagents involved in grape bud break and their effects on different metabolites and related gene expression during winter. Front. Plant Sci. 2017, 8, 1340. [CrossRef]

19. Fernandez, E.; Cuneo, I.F.; Luedeling, E.; Alvarado, L.; Farias, D.; Saa, S. Starch and hexoses concentrations as physiological markers in dormancy progression of sweet cherry twigs. Trees 2019, 33, 1187-1201. [CrossRef]

20. Ben Mohamed, H.; Vadel, A.M.; Geuns, J.M.C.; Khemira, H. Carbohydrate changes during dormancy release in Superior Seedless grapevine cuttings following hydrogen cyanamide treatment. Sci. Hortic. 2012, 140, 19-25. [CrossRef]

21. Matsoukas, I.G. Interplay between sugar and hormone signaling pathways modulate floral signal transduction. Front. Genet. 2014, 5, 218. [CrossRef] [PubMed]

22. Tang, L.; Chhajed, S.; Vashisth, T.; Olmstead, M.A.; Olmstead, J.W.; Colquhoun, T.A. Transcriptomic study of early responses to the bud dormancy-breaking agent hydrogen cyanamide in 'TropicBeauty' peach. J. Am. Soc. Hortic. Sci. 2019, 144, $244-256$. [CrossRef]

23. Zhang, T.; Yuan, Y.; Zhan, Y.; Cao, X.; Liu, C.; Zhang, Y.; Gai, S. Metabolomics analysis reveals Embden Meyerhof Parnas pathway activation and flavonoids accumulation during dormancy transition in tree peony. BMC Plant Biol. 2020, 20, 484. [CrossRef] [PubMed]

24. Michailidis, M.; Karagiannis, E.; Tanou, G.; Sarrou, E.; Adamakis, I.-D.; Karamanoli, K.; Martens, S.; Molassiotis, A. Metabolic mechanisms underpinning vegetative bud dormancy release and shoot development in sweet cherry. Environ. Exp. Bot. 2018, 155, 1-11. [CrossRef]

25. Horikoshi, H.M.; Sekozawa, Y.; Kobayashi, M.; Saito, K.; Kusano, M.; Sugaya, S. Metabolomics analysis of 'Housui' Japanese pear flower buds during endodormancy reveals metabolic suppression by thermal fluctuation. Plant Physiol. Biochem. 2018, 126, 134-141. [CrossRef] [PubMed]

26. Hildebrandt, T.M.; Nunes Nesi, A.; Araujo, W.L.; Braun, H.P. Amino acid catabolism in plants. Mol. Plant 2015, 8, 1563-1579. [CrossRef] [PubMed]

27. Yang, Q.; Zhao, D.; Liu, Q. Connections between amino acid metabolisms in plants: Lysine as an example. Front. Plant Sci. 2020, 11, 928. [CrossRef]

28. Götz, K.-P.; Chmielewski, F.-M.; Gödeke, K.; Wolf, K.; Jander, E.; Sievers, S.; Homann, T.; Huschek, G.; Rawel, H.M. Assessment of amino acids during winter rest and ontogenetic development in sweet cherry buds (Prunus avium L.). Sci. Hortic. 2017, 222, 102-110. [CrossRef]

29. Galili, G.; Amir, R.; Fernie, A.R. The regulation of essential amino acid synthesis and accumulation in plants. Annu. Rev. Plant Biol. 2016, 67, 153-178. [CrossRef]

30. Qiu, X.M.; Sun, Y.Y.; Ye, X.Y.; Li, Z.G. Signaling role of glutamate in plants. Front. Plant Sci. 2019, 10, 1743. [CrossRef]

31. Kirma, M.; Araujo, W.L.; Fernie, A.R.; Galili, G. The multifaceted role of aspartate-family amino acids in plant metabolism. J. Exp. Bot. 2012, 63, 4995-5001. [CrossRef] [PubMed]

32. Xu, H.; Curtis, T.Y.; Powers, S.J.; Raffan, S.; Gao, R.; Huang, J.; Heiner, M.; Gilbert, D.R.; Halford, N.G. Genomic, biochemical, and modeling analyses of asparagine synthetases from wheat. Front. Plant Sci. 2017, 8, 2237. [CrossRef] [PubMed]

33. Xing, A.; Last, R.L. A regulatory hierarchy of the arabidopsis branched-chain amino acid metabolic network. Plant Cell 2017, 29, 1480-1499. [CrossRef] [PubMed]

34. Peng, C.; Uygun, S.; Shiu, S.H.; Last, R.L. The impact of the branched-chain ketoacid dehydrogenase complex on amino acid homeostasis in Arabidopsis. Plant Physiol. 2015, 169, 1807-1820. [CrossRef] [PubMed]

35. Ros, R.; Munoz-Bertomeu, J.; Krueger, S. Serine in plants: Biosynthesis, metabolism, and functions. Trends Plant Sci. 2014, 19, 564-569. [CrossRef]

36. Timm, S.; Florian, A.; Wittmiss, M.; Jahnke, K.; Hagemann, M.; Fernie, A.R.; Bauwe, H. Serine acts as a metabolic signal for the transcriptional control of photorespiration-related genes in Arabidopsis. Plant Physiol. 2013, 162, 379-389. [CrossRef]

37. Maeda, H.; Dudareva, N. The shikimate pathway and aromatic amino acid biosynthesis in plants. Annu. Rev. Plant Biol. 2012, 63, 73-105. [CrossRef]

38. Batista-Silva, W.; Heinemann, B.; Rugen, N.; Nunes-Nesi, A.; Araujo, W.L.; Braun, H.P.; Hildebrandt, T.M. The role of amino acid metabolism during abiotic stress release. Plant Cell Environ. 2019, 42, 1630-1644. [CrossRef]

39. Savoi, S.; Wong, D.C.; Arapitsas, P.; Miculan, M.; Bucchetti, B.; Peterlunger, E.; Fait, A.; Mattivi, F.; Castellarin, S.D. Transcriptome and metabolite profiling reveals that prolonged drought modulates the phenylpropanoid and terpenoid pathway in white grapes (Vitis vinifera L.). BMC Plant Biol. 2016, 16, 67. [CrossRef]

40. Orrantia-Araujo, M.A.; Martínez-Téllez, M.Á.; Rivera-Domínguez, M.; Hernández-Oñate, M.Á.; Vargas-Arispuro, I. Changes in the endogenous content and gene expression of salicylic acid correlate with grapevine bud dormancy release. Plant Growth Regul. 2020. [CrossRef] 
41. Beauvieux, R.; Wenden, B.; Dirlewanger, E. Bud dormancy in perennial fruit tree species: A pivotal role for oxidative cues. Front. Plant Sci. 2018, 9, 657. [CrossRef] [PubMed]

42. Mazzitelli, L.; Hancock, R.D.; Haupt, S.; Walker, P.G.; Pont, S.D.; McNicol, J.; Cardle, L.; Morris, J.; Viola, R.; Brennan, R.; et al. Co-ordinated gene expression during phases of dormancy release in raspberry (Rubus idaeus L.) buds. J. Exp. Bot. 2007, 58, 1035-1045. [CrossRef] [PubMed]

43. Khalil-Ur-Rehman, M.; Wang, W.; Zheng, H.; Faheem, M.; Iqbal, S.; Shen, Z.G.; Tao, J. Role of hydrogen cyanamide (HC) in grape bud dormancy release: Proteomic approach. 3 Biotech 2020, 10, 229. [CrossRef] [PubMed]

44. Pérez, F.J.; Vergara, R.; Or, E. On the mechanism of dormancy release in grapevine buds: A comparative study between hydrogen cyanamide and sodium azide. Plant Growth Regul. 2009, 59, 145-152. [CrossRef] 